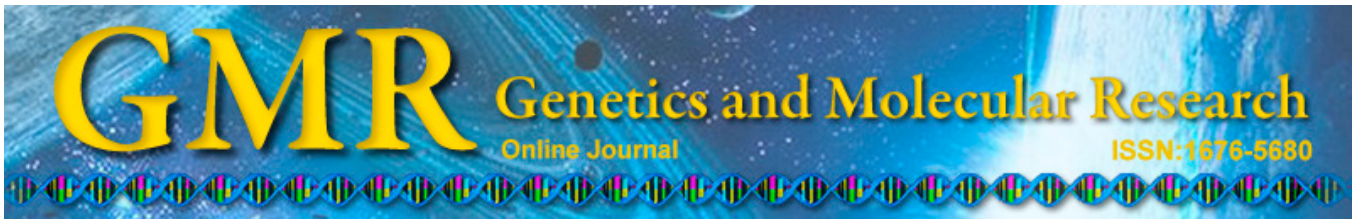

\title{
NEDD4-1 and PTEN expression in keloid scarring
}

\author{
P.F. Sang, H. Wang, M. Wang, C. Hu, J.S. Zhang, X.J. Li and F. Zhu \\ Department of Plastic Surgery, \\ First Affiliated Hospital of Anhui Medical University, Hefei, China \\ Corresponding author: F. Zhu \\ E-mail: zhufei_1@yeah.net
}

Genet. Mol. Res. 14 (4): 13467-13475 (2015)

Received January 9, 2015

Accepted June 8, 2015

Published October 28, 2015

DOI http://dx.doi.org/10.4238/2015.October.28.7

\begin{abstract}
Keloid scarring remains a major problem in plastic surgery. The aim of this study was to determine the expression of the PTEN tumor suppressor and NEDD4-1 genes in keloid tissue and explore their effect on the formation of such scarring. Twenty keloid patients were enrolled in the study and underwent surgical removal of keloid tissue. No patient had received chemotherapy and/or radiotherapy prior to treatment. PTEN and NEDD4-1 mRNA expression was detected by reverse transcription PCR, while PTEN protein expression was assessed using immunohistochemistry. Our results showed that levels of PTEN were significantly diminished in keloid samples $(\mathrm{P}<0.05)$, whereas those of NEDD4-1 did not significantly differ between keloid tissue and normal skin $(\mathrm{P}>0.05)$. Furthermore, we found that NEDD4-1 expression is high and inversely correlated with that of PTEN in keloids. Our results suggest that the PTEN/PI3K/AKT pathway may play an important role in keloid formation and reduces PTEN expression in such tissue. Finally, although NEDD4-1 has previously been identified as a factor in keloid susceptibility, and the protein for which it encodes is known to degrade PTEN by catalyzing its polyubiquitylation, the detailed mechanism behind its involvement in keloid formation needs to be further studied.
\end{abstract}

Key words: NEDD4-1; PTEN; Pathological scar; Keloid 


\section{INTRODUCTION}

The process of wound healing in humans is a complex one that relies on the interplay between multiple cell types, cytokines, and proteins. Under usual circumstances, this process results in a scar that over time flattens and softens, typically leaving little long-term impact. Aberrancies in this process exist, however, leading to various forms of excessive scarring such as hypertrophic scars and keloids (Naylor and Brissett, 2012).

Keloids are fibroproliferative tumors with no known malignant potential that occur in locations of dermal trauma or disruption. They are characterized by their extension beyond the confines of the original injury and often present with pain and pruritus. Additionally, these growths may result in cosmetic deformities and contribute to significant emotional distress (Naylor and Brissett, 2012). The exact etiology is unknown, but significant research efforts have been made (Xiaoxue et al., 2014). These investigations have demonstrated that several genetic alterations are involved in the carcinogenetic formation process and it has been suggested that a subset of these abnormalities concern the genes phosphatase and tensin homolog (PTEN) and neural precursor cell expressed, developmentally downregulated 4-1 (NEDD4-1).

The protein PTEN is a dual-specificity phosphatase that acts as a negative regulator of the phosphoinositide 3-kinase (PI3K)/v-akt murine thymoma viral oncogene homolog (AKT)/ mechanistic target of rapamycin (mTOR) pathway (Liao et al., 2010; Kulkarni et al., 2011; Paterno et al., 2011), thus controlling a variety of processes related to cell survival, proliferation, and growth (Wang et al., 2008). The loss of PTEN function due to deletion, mutation, methylation, or decreased expression has been observed in human cancers (Ma et al., 2009; Miller et al., 2009; Yang et al., 2010) and some fibrotic diseases (White et al., 2003; Takashima et al., 2009). In addition, in idiopathic pulmonary fibrosis and liver fibrosis, both mRNA and protein levels of PTEN are suppressed (White et al., 2006; Larsson et al., 2008). Although keloids are not malignant tumors, the fibroblasts involved exhibit malignant features such as excessive proliferation, resistance to apoptosis, and atypical differentiation (Lim et al., 2006), and the mechanism behind their formation is not yet fully understood (Atiyeh, 2007). We therefore hypothesized that PTEN levels should be diminished in keloid pathogenesis.

In a genome-wide association study, Nakashima et al. (2010) identified NEDD4 as a keloid susceptibility gene in the Japanese population. More recently, Zhu et al. (2013) used the Sequenom MassARRAY system to determine that NEDD4 is also significantly associated with keloid susceptibility in the Han Chinese population. Recent research has shown that through the PI3K/AKT pathway, NEDD4 may promote fibroblast proliferation and invasiveness and cause loss of cellular contact inhibition (Akca et al., 2011). p27 protein, a cyclin-dependent kinase inhibitor, plays an important role in cell contact inhibition by arresting the cell cycle, while the highly expressed NEDD4 gene may induce the transfer of p27 from the nucleus to the cytoplasm and promote its degradation (Liu et al., 2002). NEDD4 may also promote the excessive expression and accumulation in the extracellular matrix of fibroblast protein and type I collagen (Liu et al., 2013). Taken together, these results suggest that NEDD4 may promote keloid formation and development. Furthermore, NEDD4 has been found to effect the accumulation of $\beta$-catenin in the cytoplasm of fibroblasts, and to activate its signal transduction pathway. According to the same report, mass accumulation of $\beta$-catenin is found in keloid tissue (Sato, 2006; Chung et al., 2011). It can therefore be inferred from this that increased expression of NEDD4 may be implicated in the formation and development of keloids. NEDD4- 
1 is one of the most important NEDD4 isoforms. It was originally identified as an E3 ligase that ubiquitylates PTEN, and regulates the apoptosis and proliferation of tumor cells (Wang et al., 2007; Kwak et al., 2010). We therefore hypothesized that an inverse correlation between PTEN and NEDD4-1 levels may be implicated in keloid pathogenesis.

In the present study, the mRNA levels of PTEN and NEDD4-1 in keloids and hypertrophic scars were measured by reverse transcription polymerase chain reaction (RT-PCR), and the expression of PTEN protein was assessed using immunohistochemistry. Our findings may provide a new target for the prevention and cure of keloid formation.

\section{MATERIAL AND METHODS}

\section{Tissue specimens}

Keloid tissues were obtained from 20 people (16 men and 4 women, 12-47 years old). No local infection or ulceration was evident and no study subject had been treated with glucocorticosteroids or radiotherapy. Twenty age- and site-matched normal skin (NS) specimens were obtained from donors during scar resection. Experimental protocols were approved by the hospital Ethics Committee and written informed consent was obtained from each donor.

\section{RT-PCR}

Total RNA was extracted with TRIzol (Invitrogen, Carlsbad, CA, USA), following the manufacturer protocol. Primers were designed using Primer Premier 5.0 (PREMIER Biosoft International, Palo Alto, CA, USA) and synthesized by the Shanghai Shenggong Genomics Institute (Shanghai, China). Primer sequences were as follows: PTEN forward: 5'-CAGAGCGAGGGGCATCAG-3', reverse: 5'-GCAGGAAATCCCATAGCAATAA-3', (238-bp product); NEDD4-1 forward: 5'-GCATGTTTGCCATCCTCCCA-3', reverse: 5'-AGCCAGGCTTGCAAGAATTAG-3', (295-bp product); and glyceraldehyde 3-phosphate dehydrogenase $(G A P D H)$ forward: 5'-AGATCATCAGCAATGCCTCCTG-3', and reverse: 5'-ATGGCATGGACTGTGGTCATG-3', (109-bp product). Each PCR was performed as follows: denaturation at $94^{\circ} \mathrm{C}$ for $5 \mathrm{~min}$, then 35 cycles of $94^{\circ} \mathrm{C}$ for $30 \mathrm{~s}, 55^{\circ} \mathrm{C}$ for $30 \mathrm{~s}$, and $72^{\circ} \mathrm{C}$ for $30 \mathrm{~s}$. After 35 cycles, products of the exponential phase of the reaction were assessed to allow semi-quantitative comparison of complementary DNA synthesized from identical reactions. Amplification products were analyzed on a $2 \%$ agarose gel and visualized by ethidium bromide staining. The gray values of each bands in RT-PCR gel were analyzed by the Image J 2.1.4.7 software.

\section{Immunohistochemistry}

Keloid and NS tissues were fixed with formalin and embedded in paraffin following routine histological practices. A primary antibody against PTEN was used at a dilution of 1 in 200 (Santa Cruz Biotechnology, Santa Cruz, CA, USA). To detect antibody-bound cells, an avidin/biotinylated enzyme complex was used (Vectastain $\mathrm{ABC}$ kit; Vector Laboratories, Burlingame, CA, USA). Tissues were examined under an Olympus microscope (Olympus, Tokyo, Japan) and images were taken using a SPOT digital microscope camera (Diagnostic Instruments, Sterling Heights, MI, USA). 


\section{Data analysis}

Data are reported as means $\pm \mathrm{SE}$ and were analyzed using the Shapiro-Wilk test. Statistical significance was determined using SPSS 15.0 (SPSS Inc., Chicago, IL, USA). Pearson's correlation test was also used to examine relationships, and $\mathrm{P}$ values equal to or less than 0.05 were considered to be statistically significant.

\section{RESULTS}

\section{PTEN expression in keloid and NS tissues}

Results were obtained for all cases. By semi-quantitative RT-PCR, we found that PTEN mRNA expression was markedly reduced in keloid tissue compared to that of NS (representative samples are shown in Figure 1A). Quantitative analysis from 20 patients also indicated that PTEN mRNA levels in keloid tissues were significantly lower than those in NS samples (Figure 1B, P $<0.01$ ). In addition, immunohistochemical examination demonstrated strong PTEN immunoreactivity in both the cytoplasm and nuclei of epithelial and dermal fibroblasts in NS tissues (Figure 1C), while only a weak signal, or an absence thereof, was detected in keloid samples (Figure 1D). This suggests that PTEN protein expression is diminished in keloid tissue.

\section{NEDD4-1 expression in keloid and NS tissues}

Using semi-quantitative RT-PCR, we found that NEDD4-1 mRNA expression did not significantly differ between keloid and NS specimens (representative samples are shown in Figure 1E). Quantitative analysis from 20 patients also indicated that NEDD4-1 mRNA levels in keloid tissues were not significantly lower than those in NS samples (Figure $1 \mathrm{~F}, \mathrm{P}>0.05$.)

\section{NEDD4-1 expression is high and inversely correlated with that of PTEN in keloid tissue}

Although overall NEDD4-1 expression was not found to be significantly different between in keloid tissues and NS, this gene has previously been identified as a factor resulting in keloid susceptibility, and the encoded protein is a ubiquitin ligase capable of degrading PTEN. Therefore, to investigate the correlation between PTEN and NEDD4-1 levels in keloid tissues (Figure 2, lane 1), hypertrophic tissues (Figure 2, lane 2) and normal tissues (Figure 2, lane 3), RT-PCR analysis was performed using specific primers against these two transcripts (Figure 2). A significant inverse correlation between PTEN and NEDD4-1 expression was confirmed.

In summary, quantitative analysis of samples from 20 patients showed that the level of PTEN was significantly lower in keloid tissue than in NS. 
$\mathbf{A}$

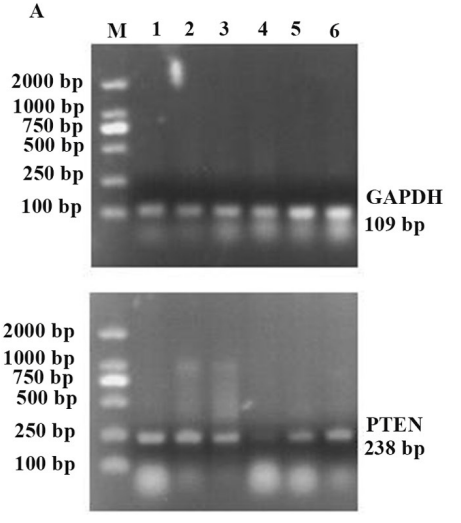

C

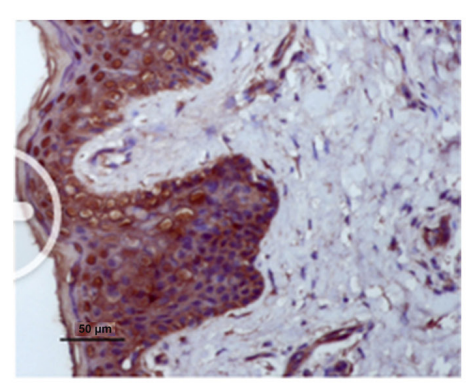

E

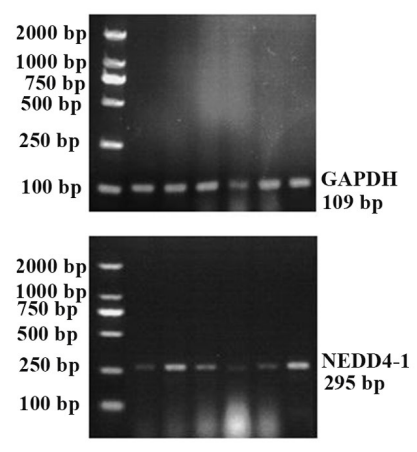

B

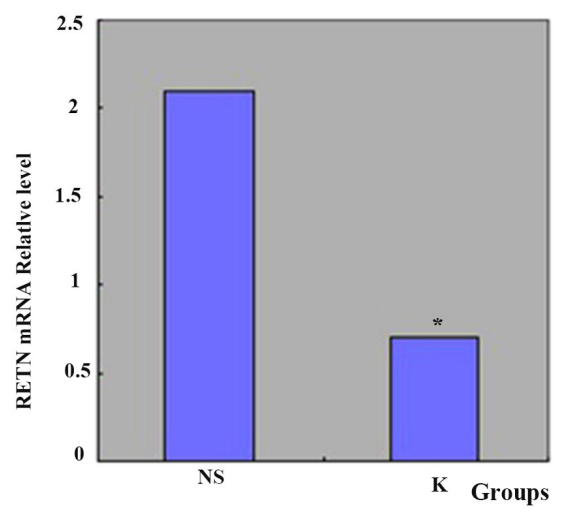

D

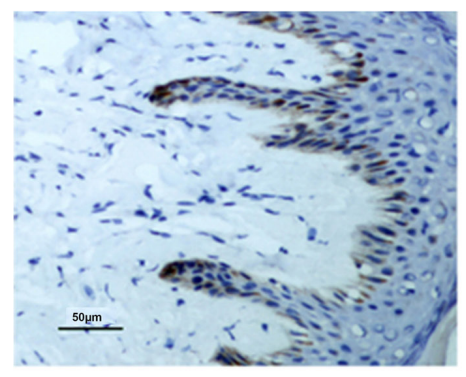

F

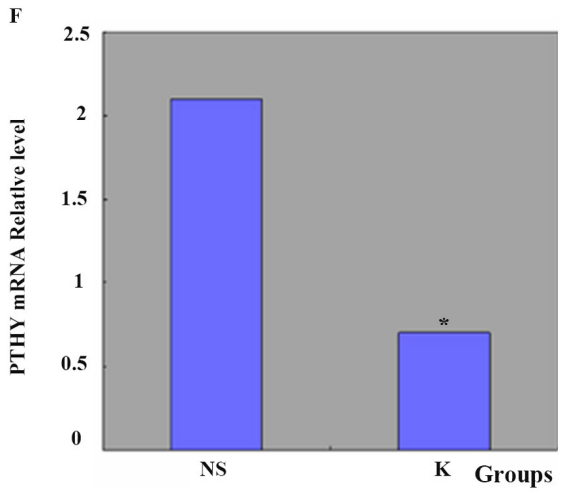

Figure 1. PTEN and NEDD4-1 expression in tissues. A. Reverse transcription polymerase chain reaction (RT-PCR) analysis of PTEN mRNA in tissues from two patients. Lane M, DNA marker. B. Semi-quantitative evaluation of PTEN mRNA level in two tissue types. Data are reported as the ratio of PTEN expression to that of GAPDH. NS, normal skin; K, keloid tissue. $* \mathrm{P}<0.05$. C. Immunohistochemical staining of PTEN in NS. Strong PTEN immunoreactivity (brown particles) was observed in the cytoplasm of epithelial and dermal fibroblasts in NS. Bar $=50 \mu \mathrm{m}$. D. Immunohistochemical staining of PTEN in keloid tissue, showing very little immunoreactivity. Brown particles indicate a strong reaction. E. RT-PCR analysis of NEDD4-1 mRNA in tissues from two patients. Lane M, DNA marker. F. Semi-quantitative evaluation of NEDD4-1 mRNA level in two tissue types. Data are reported as the ratio of NEDD4-1 expression to that of GAPDH. NS, normal skin; K, keloid tissue. *P $>0.05$. 

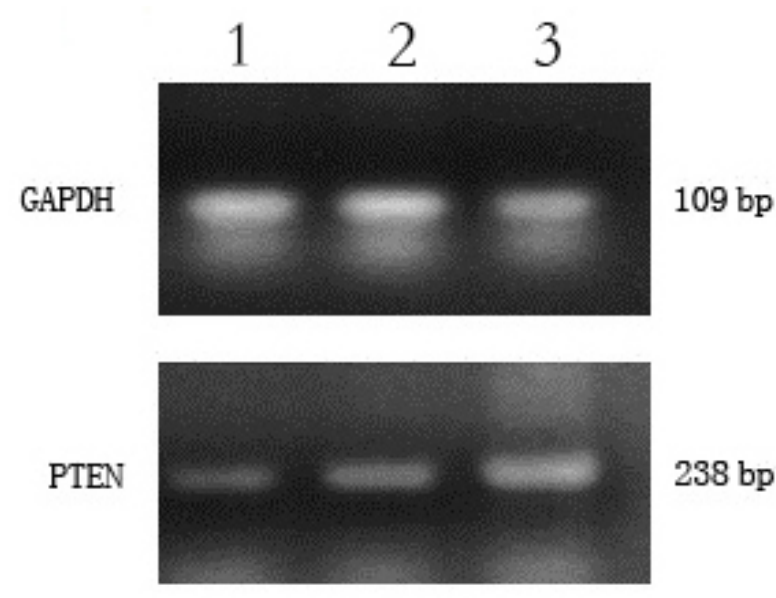

$238 \mathrm{bp}$

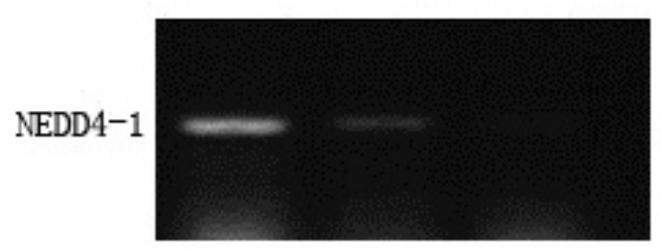

$295 \mathrm{bp}$

Figure 2. Reverse transcription polymerase chain reaction analysis of PTEN and NEDD4-1 expression in keloid tissues (lane 1), hypertrophic tissues (lane 2) and normal tissues (lane 3). GAPDH served as a loading control.

\section{DISCUSSION}

Keloids (Xiaoxue et al., 2008) are benign skin tumors that may appear after wound healing in genetically predisposed patients. They are characterized by excessive proliferation of fibroblast cells and an overabundance of collagen at the site of a healed injury (Al-Attar et al., 2006), and can invade skin beyond the boundaries of the original wound, with no spontaneous regression (Fujiwara et al., 2005).

It has been generally accepted that loss of PTEN function with subsequent activation of the PI3K/AKT signaling pathway contributes to carcinogenesis and fibrotic diseases (Li et al., 2012), and PTEN has been proposed as an effective anti-fibrosis target (Xia et al., 2008; Parapuram et al., 2011).

In the present study, we found that PTEN mRNA expression was significantly downregulated in keloid tissue compared to NS. Furthermore, immunohistochemical analyses revealed that the level of PTEN protein was also lower in samples derived from keloid specimens. These results suggest that the expression of PTEN is downregulated at both transcriptional and translational levels in keloids, and abnormal PTEN expression is involved in their pathogenesis. In conclusion, loss of PTEN function may play an important role in the development of keloid tissue.

The ubiquitin-mediated proteasomal pathway is an important mechanism that regulates protein stability. Recently, Wang et al. (2007) found that the level of PTEN protein is 
regulated by ubiquitin-mediated proteosomal degradation (Figure 1), and identified its ubiquitin ligase as NEDD4-1. NEDD4-1 degrades PTEN protein by catalyzing PTEN polyubiquitylation. In cells, overexpression of NEDD4-1 promotes Kirsten rat sarcoma viral oncogene homolog (K-RAS)-induced oncogenic cellular transformation in a PTEN-dependent manner. Moreover, in human urinary bladder cancers, an inverse relationship between NEDD4-1 and PTEN levels has been observed (Wang et al., 2007). Together, these data strongly suggest that NEDD4-1 demonstrates oncogenic activity by suppressing PTEN function. To date, the expression and cellular localization of NEDD4-1 has not been established in keloid scarring. To extend our knowledge regarding the role of NEDD4-1 in this process, we compared its expression in keloid and normal tissue.

Although expressed in both tissue types, no significant difference in the level of NEDD4-1 was observed between keloid and NS samples. In addition, we also found that NEDD4-1 expression levels were inversely correlated with those of PTEN in keloid tissues, indicating its proto-oncogenic properties.

In addition to its oncogenic function, NEDD4-1 is believed to play a role in tumor suppression by catalyzing PTEN monoubiquitylation, leading to PTEN nuclear import (Trotman et al., 2007), where it plays a crucial role in maintaining chromosomal integrity (Shen et al., 2007; Trotman et al., 2007).

NEDD4-1 regulates PTEN function, and may play a crucial role in the development of keloids. However, expression of NEDD4-1 mRNA was not seen to differ significantly between keloid and normal tissue in this study. There are several possible reasons behind this finding. First, recent research into the genetics of keloid development has shown that this condition is a polygenic disease, and may be influenced by the additive effect of several susceptibility genes, as well as environmental factors. Taken on its own, a single susceptibility gene will tend to have a minor impact and may not affect the overall process of keloid development, as appears to be the case with NEDD4-1. Second, this result may be related to selection bias due to a relatively small sample size. Third, the formation of keloids may be caused by mutations in the NEDD4-1 gene that do not affect the level of protein expressed, and thus in that regard, significant differences between keloid and normal tissue would not be detected.

In humans, the NEDD4- 1 gene is expressed in both normal skin tissue and keloids. In terms of its being implicated in susceptibility, the role of NEDD4-1 in the occurrence and development of keloid tissue requires further study. In the future, two aspects need to be thoroughly addressed. First, experimental error and selection bias can be reduced by increasing the sample size. Second, as NEDD4-1 sequence variations caused by genetic mutations presumably exist, any differences in this respect can be contrasted by experimental detection and database search and alignment. In addition, advanced exome sequencing technology may present a new method for further inquiry into keloid susceptibility genes.

\section{Conflicts of interest}

The authors declare no conflict of interest.

\section{ACKNOWLEDGMENTS}

Research partially supported by Chinese Han keloid susceptibility gene-related research projects (\#KJ2014A108). 


\section{REFERENCES}

Akca H, Demiray A, Tokgun O and Yokota J (2011). Invasiveness and anchorage independent growth ability augmented by PTEN inactivation through the PI3K/AKT/NFkB pathway in lung cancer cells. Lung Cancer 73: 302-309.

Al-Attar A, Mess S, Thomassen IM, Kauffman CL, et al. (2006). Keloid pathogenesis and treatment. Plast. Reconstr. Surg. 117: 286-300.

Atiyeh BS (2007). Nonsurgical management of hypertrophic scars: evidence-based therapies, standard practices, and emerging methods. Aesthetic Plast. Surg. 31: 468-492.

Chung S, Nakashima M, Zembutsu H and Nakamura Y (2011). Possible involvement of NEDD4 in keloid formation; its critical role in fibroblast proliferation and collagen production. Proc. Jpn. Acad. Ser. B Phys. Biol. Sci. 87: 563-573.

Fujiwara M, Muragaki Y and Ooshima A (2005). Keloid-derived fibroblasts show increased secretion of factors involved in collagen turnover and depend on matrix metalloproteinase for migration. Br. J. Dermatol. 153: 295-300.

Kulkarni AA, Thatcher TH, Olsen KC, Maggirwar SB, et al. (2011). PPAR-gamma ligands repress TGF $\beta$-induced myofibroblast differentiation by targeting the PI3K/Akt pathway: implications for therapy of fibrosis. PLoS One 6: e15909.

Kwak YD, Wang B, Pan W, Xu H, et al. (2010). Functional interaction of phosphatase and tensin homologue (PTEN) with the E3 ligase NEDD4-1 during neuronal response to zinc. J. Biol. Chem. 285: 9847-9857.

Larsson O, Diebold D, Fan D, Peterson M, et al. (2008). Fibrotic myofibroblasts manifest genome-wide derangements of translational control. PLoS One 3: e3220.

Li T, Yang Y, Li X, Xu C, et al. (2012). EGFR- and AKT-mediated reduction in PTEN expression contributes to tyrphostin resistance and is reversed by mTOR inhibition in endometrial cancer cells. Mol. Cell. Biochem. 361: 19-29.

Liao WT, Yu HS, Arbiser JL, Hong CH, et al. (2010). Enhanced MCP-1 release by Keloid CD14+ cells augments fibroblast proliferation: role of MCP-1 and Akt pathway in keloids. Exp. Dermatol. 19: e142-e150.

Lim CP, Phan TT, Lim IJ and Cao X (2006). Stat3 contributes to keloid pathogenesis via promoting collagen production, cell proliferation and migration. Oncogene 25: 5416-5425.

Liu S, Parapuram SK and Leask A (2013). Fibrosis caused by loss of PTEN expression in mouse fibroblasts is crucially dependent on CCN2. Arthritis Rheum. 65: 2940-2944.

Liu W, Asa SL and Ezzat S (2002). Vitamin D and its analog EB1089 induce p27 accumulation and diminish association of p27 with Skp2 independent of PTEN in pituitary corticotroph cells. Brain Pathol. 12: 412-419.

Ma J, Sawai H, Ochi N, Matsuo Y, et al. (2009). PTEN regulates angiogenesis through PI3K/Akt/VEGF signaling pathway in human pancreatic cancer cells. Mol. Cell. Biochem. 331: 161-171.

Miller KA, Yeager N, Baker K, Liao XH, et al. (2009). Oncogenic Kras requires simultaneous PI3K signaling to induce ERK activation and transform thyroid epithelial cells in vivo. Cancer Res. 69: 3689-3694.

Nakashima M, Chung S, Takahashi A, Kamatani N, et al. (2010). A genome-wide association study identifies four susceptibility loci for keloid in the Japanese population. Nat. Genet. 42: 768-771.

Naylor MC and Brissett AE (2012). Current concepts in the etiology and treatment of keloids. Facial Plast. Surg. 28: 504-512.

Parapuram SK, Shi-Wen X, Elliott C, Welch ID, et al. (2011). Loss of PTEN expression by dermal fibroblasts causes skin fibrosis. J. Invest. Dermatol. 131: 1996-2003.

Paterno J, Vial IN, Wong VW, Rustad KC, et al. (2011). Akt-mediated mechanotransduction in murine fibroblasts during hypertrophic scar formation. Wound Repair Regen. 19: 49-58.

Sato M (2006). Upregulation of the Wnt/ $\beta$-catenin pathway induced by transforming growth factor- $\beta$ in hypertrophic scars and keloids. Acta Derm. Venereol. 86: 300-307.

Shen WH, Balajee AS, Wang J, Wu H, et al. (2007). Essential role for nuclear PTEN in maintaining chromosomal integrity. Cell 128: 157-170.

Takashima M, Parsons CJ, Ikejima K, Watanabe S, et al. (2009). The tumor suppressor protein PTEN inhibits rat hepatic stellate cell activation. J. Gastroenterol. 44: 847-855.

Trotman LC, Wang X, Alimonti A, Chen Z, et al. (2007). Ubiquitination regulates PTEN nuclear import and tumor suppression. Cell 128: 141-156.

White ES, Thannickal VJ, Carskadon SL, Dickie EG, et al. (2003). Integrin alpha4betal regulates migration across basement membranes by lung fibroblasts: a role for phosphatase and tensin homologue deleted on chromosome 10 . Am. J. Respir. Crit. Care Med. 168: 436-442.

White ES, Atrasz RG, Hu B, Phan SH, et al. (2006). Negative regulation of myofibroblast differentiation by PTEN (Phosphatase and Tensin Homolog Deleted on chromosome 10). Am. J. Respir. Crit. Care Med. 173: 112-121.

Wang X, Trotman LC, Koppie T, Alimonti A, et al. (2007). NEDD4-1 is a proto-oncogenic ubiquitin ligase for PTEN. Cell 128: 129-139. 
Wang X, Shi Y, Wang J, Huang G, et al. (2008). Crucial role of the C-terminus of PTEN in antagonizing NEDD4-1mediated PTEN ubiquitination and degradation. Biochem. J. 414: 221-229.

Xia H, Diebold D, Nho R, Perlman D, et al. (2008). Pathological integrin signaling enhances proliferation of primary lung fibroblasts from patients with idiopathic pulmonary fibrosis. J. Exp. Med. 205: 1659-1672.

Xiaoxue W, Xi C and Zhibo X (2014). Effects of botulinum toxin type A on expression of genes in keloid fibroblasts. Aesthet. Surg. J. 34: 154-159.

Yang J, Ren Y, Wang L, Li B, et al. (2010). PTEN mutation spectrum in breast cancers and breast hyperplasia. J. Cancer Res. Clin. Oncol. 136: 1303-1311. 